Professor Dr. Thomas M.J. Möllers und Alexandra Möhring, Augsburg*

\title{
Recht und Pflicht zur richtlinienkonformen Rechtsfortbildung bei generellem Umsetzungswillen des Gesetzgebers
}

\author{
- Zugleich Besprechung von EuGH Rs. C-404/06 (Quelle AG/Bundesverband \\ der Verbraucherzentralen und Verbraucherverbände) -
} \begin{abstract}
widerspricht bei Verbraucherverträgen der Abs. 4 BGB im Wege der richtlinienkonformen vereinbaren lässt.

\section{Die doppelte Bedeutsamkeit der Entscheidung}

Dem Urteil des EuGH (JZ 2008, 942, in diesem Heft) zufolge Nutzungsersatzanspruch bei Rückgabe fehlerhafter Ware gemäß §§ 439 Abs. 4 i. V.m. 346 Abs. 1 a. E., Abs. 2 Nr. 1 BGB der Verbrauchsgüterkaufrichtlinie. Der Beitrag zeigt, dass eine teleologische Reduktion der Verweisungsnorm des $\S 439$

Rechtsfortbildung sich durchaus mit der nationalen Dogmatik

Die Entscheidung des $E u G H$ ist von besonderer Relevanz: Sie ist die erste Entscheidung zur wichtigsten Richtlinie im Bereich des Verbraucherschutzes, der Verbrauchsgüterkaufrichtlinie 1999/44/EG (nachfolgend „RiL“). Sie ergeht nach einer Vorlage durch den $B G H^{1} \mathrm{zu}$ der wichtigen Frage, ob nach deutschem Recht bei Nachlieferung einer mangelhaften Sache dem Verkäufer weiterhin ein Nutzungsersatzanspruch zusteht. Der EuGH stellte fest, dass das BGB in der Fassung der Schuldrechtsmodernisierung gegen europäisches Recht verstößt. ${ }^{2}$ Dies hat weitreichende, ökonomische Auswirkungen für den Verkäufer und die Hersteller, aber auch für den Verbraucher.

Das Besondere an dieser Entscheidung ist vor allem die interessante Folgefrage, wie der VIII. Senat des $B G H$ die Vorgaben des EuGH berücksichtigen wird. Er hatte angedeutet, eine richtlinienkonforme Auslegung sei nicht möglich, da einer solchen Auslegung neben dem eindeutigen Wortlaut insbesondere der in den Gesetzesmaterialien zum Ausdruck gebrachte eindeutige Wille des Gesetzgebers entgegenstehe. ${ }^{3}$ In der Literatur wurde behauptet, dass die Bundesrepublik Deutschland wegen der unzureichenden Umsetzung der RiL deshalb Schadensersatz aufgrund des gemeinschaftsrechtlichen Staatshaftungsanspruchs zu leisten hätte. ${ }^{4}$

\footnotetext{
* Prof. Dr. Thomas M.J. Möllers ist Inhaber des Lehrstuhls für Bürgerliches Recht, Europarecht, Wirtschaftsrecht, Internationales Privatrecht und Rechtsvergleichung an der Universität Augsburg; Alexandra Möhring ist wiss. Assistentin am Lehrstuhl.

$1 B G H$ VIII ZR 200/05 - Vorlagebeschluss Quelle = NJW 2006, 3200.

2 EuGH Rs. C-404/06 - Quelle = JZ 2008, 942 = NJW 2008, $1433 \mathrm{~m}$. Anm. Staudinger ZJS 2008, 309 f.; Fischinger EuZW 2008, 310; Schulze GPR 2008, 128; Faust JuS 2008, 652; Felling MDR 2008, 733; Herrer/Tomasic BB 2008, 1245; Herresthal NJW 2008, 2475.

$3 B G H$ NJW 2006, 3200, 3201 Rn. 12, 15 - Vorlagebeschluss Quelle.

4 Einen solchen bejahend Lorenz NJW 2007, 3203; ders. NJW 2008, 1, 6;

Schürnbrand JZ 2007, 910, 917; Hummel EuZW 2007, 268, 271; Fischinger
}

\section{Zulässigkeit der Vorlagefrage}

Die Frage, ob die Vorlage an den $E u G H$ zulässig ist, war im Vorfeld bereits heftig diskutiert worden. So wurde etwa behauptet, der $B G H$ habe die Vorfrage gestellt, um einen Hinweis auf die unmittelbare Geltung der RiL auch zwischen Privaten $\mathrm{zu}$ erlangen. ${ }^{5}$ Allerdings war die Vorlagefrage eindeutig nur auf die Auslegung des Art. 3 Abs. 2 bis 4 RiL gerichtet. ${ }^{6}$ Folgerichtig hatte auch der EuGH die unmittelbare Wirkung der RiL mit keinem Wort erwähnt. ${ }^{7}$ Von anderer Seite wurde die Zulässigkeit der Vorlagefrage in Zweifel gezogen: Wenn nach Ansicht des VIII. Senates des $B G H$ eine Auslegung gegen Wortlaut und Wille des Gesetzgebers und damit eine richtlinienkonforme Rechtsfortbildung nicht möglich sei, sei nach der „Logik“ des vorliegenden Beschlusses die Vorlage an den EuGH mangels Entscheidungserheblichkeit „ebenso unnötig wie unzulässig“. 8

Der VIII. Senat war allerdings aus dreierlei Gründen gut beraten, die Frage der Europarechtskonformität des nationalen Rechts durch den $E u G H$ prüfen zu lassen: Erstens dient, wie der EuGH zu Recht betonte, die Beantwortung von Vorlagefragen der einheitlichen Anwendung des Gemeinschaftsrechts. ${ }^{9}$ Zweitens ist die Frage, in welchem Umfang nationale Gerichte die Vorgaben des EuGH in das nationale Recht umsetzen können, der Entscheidung des EuGH logisch nachrangig. Die Umsetzung der Entscheidung des $E u G H$ durch das nationale Gericht als zweiter Schritt kann deshalb den $E u G H$ nicht daran hindern, in einem ersten Schritt die Auslegung einer Richtlinie verbindlich zu klä-

$\overline{\text { EuZW 2008 }}, 310,312$; vorsichtiger und im Ergebnis verneinend Staudinger ZJS 2008, 309, 311.

5 Hummel EuZW 2007, 268, 269.

$6 B G H$ NJW 2006, 3200, 3202 Rn. 26 sowie Leitsatz, wiedergegeben in $E u G H$ Rs. C-404/06 - Quelle = JZ 2008, 942 Rn. 16. Dieser Satz ist ein Paradebeispiel für fragwürdiges Juristendeutsch - nur eingeweihten Juristen ist er nach mehrmaligem Wiederholen verständlich. Hierzu vertiefend Möllers, Juristische Arbeitstechnik, 4. Aufl. 2008, \$6.

7 Im Übrigen lehnt der EuGH die unmittelbare Drittwirkung von Richtlinien zwischen Privaten in ständiger Rechtsprechung ab, s. grundlegend EuGH Rs. C-152/84, Slg. 1986, 723 Rn. 48 - Marshall; Rs. C- 91/92, Slg. 1994, I-3325 Rn. 20, 24 - Faccini Dori = JZ 1995, 149 mit Anm. Heß; verb. Rs. C-397/01 bis C-403/01, Slg. 2004, I-8835 Rn. 108 - Pfeiffer. Etwas anderes folgt auch nicht aus der Entscheidung EuGH Rs. C-144/04, Slg. 2005, I-9981 - Mangold, die sich auf einen Verstoß gegen den Gleichheitsgrundsatz des Primärrechts bezog.

8 Wörtlich Lorenz NJW 2006, 3202; ders. NJW 2007, 1, 6; ihm folgend $P$. Schmidt ZGS 2006, 408, 410. Franzen JZ 2003, 321, 324 für das HeiningerVerfahren. Auch die Verteidigung der Quelle AG schloss sich dieser Argumentation an. A. A. Witt NJW 2006, 3322, 3323.

9 EuGH Rs. C-404/06 - Quelle = JZ 2008, 942 Rn. 22; zustimmend Staudinger ZJS 2008, 309; Schulze GPR 2008, 128, 130. 
ren. ${ }^{10}$ Mit der Entscheidung, die Zulässigkeit der Vorlage zu bejahen, bleibt der $E u G H$ damit auch seiner Position treu, nur in engen Ausnahmefällen die Zulässigkeit des Vorabentscheidungsverfahrens zu verneinen. ${ }^{11}$ Weil die Grenzen der richtlinienkonformen Rechtsfortbildung höchst umstritten sind (s. IV.), tat der VIII. Senat gut daran, seine vorläufige Sicht einer vermeintlich unzulässigen Rechtsfortbildung contra legem nicht zu einem Zulassungshindernis zu erheben. Er hätte damit nämlich drittens das Risiko provoziert, dass eine Verletzung der Vorlagepflicht ein Vertragsverletzungsverfahren gem. Art. $226 \mathrm{f}$. EGV, eine mögliche Staatshaftung ${ }^{12}$ oder schließlich einen Verstoß gegen das Gebot des gesetzlichen Richters gem. Art. 101 Abs. 1 Satz 2 GG zur Folge gehabt hätte. Auch wenn die Voraussetzungen für solche Verstöße als recht hoch angesehen werden, ${ }^{13}$ hat das $B \operatorname{Verf} G$ einen Verstoß gegen Art. 101 Abs. 2 GG bereits dann bejaht, wenn „mögliche Gegenauffassungen zu der entscheidungserheblichen Frage des Gemeinschaftsrechts gegenüber der vom Gericht vertretenen Meinung eindeutig vorzuziehen sind“..14

\section{Die inhaltlichen Vorgaben der Quelle- Entscheidung}

\section{Unentgeltlichkeit versus deutscher Nutzungsersatzanspruch}

Die Frage, ob der Anspruch auf Nutzungsersatzanspruch des Verkäufers gegenüber dem Verbraucher gem. $\$ 439$ Abs. 4 i. V.m. $\ 346$ Abs. 1 a.E., Abs. 2 Nr. 1 BGB den Regelungen in Art.3 Abs.2-4 RiL entgegensteht, war im deutschen Schrifttum sehr umstritten. Die wohl schon damals herrschende Ansicht bejahte die Europarechtswidrigkeit des deutschen Rechts. ${ }^{15}$ Auch der $B G H$ tendierte mit dem Vorlageverfahren in diese Richtung. ${ }^{16}$

Oft war dem $E u G H$ vorgeworfen worden, seine Urteile nicht sorgfältig zu begründen. ${ }^{17}$ In der Quelle-Entscheidung nutzt der EuGH aber zahlreiche der juristischen Argumentations- und Auslegungsfiguren nahezu mustergültig: Schon der Wortlaut der Legaldefinition des Art. 3 Abs. 4 RiL lasse den Nutzungsersatz nicht zu, wie auch das Wort „insbeson-

10 EuGH Rs. C-404/06 - Quelle = JZ 2008, 942 Rn. 22. Konsequent bejaht Witt NJW 2006, 3322, 3323 deshalb auch eine Vorlagepflicht.

11 Etwa wenn der Zusammenhang mit gemeinschaftsrechtlichen Normen offensichtlich nicht gegeben war, vgl. EuGH Rs. C-119/05, Slg. 2007, I$6199-$ Lucchini $=$ JZ 2008, 141 mit Anm. Haratsch/Hensel.

$12 E u G H$ Rs. C-224/01, Slg. 2003, I-10239 Rn. $53-$ Köbler = JZ 2004, 295 mit Anm. von Danwitz; Rs. C-173/03, Slg. 2003, I-5177 Rn. 30-35, 44 - Traghetti del Mediterraneo.

$13 \mathrm{Zu}$ diesen drei Fallgruppen bei einer Verletzung der Vorlagepflicht s. Wegener, in: Callies/Ruffert, EUV/EGV, 3. Aufl. 2006, Art. 234 Rn. 30 ff.; Ehricke in: Streinz, EUV/EGV, 2003, Art. 234 Rdn. $45 \mathrm{ff}$.; Schwarze, in: Schwarze, EU-Kommentar, 2000, \$2 234 Rn. $50 \mathrm{ff}$

$14 B \operatorname{VerfG}$ NJW 2001, 1267, 1268.

15 Roth JZ 2001, 475, 489; Hoffmann ZRP 2001, 347, 349; Büdenbender, in: Dauner-Lieb/Heidel/Lepa/Ring, Anwaltskommentar, Schuldrecht, 2. Aufl. 2005, \439 Rn. 43; Gsell NJW 2003, 1969, 1973; Ball NZV 2004, 217, 221; Rott BB 2004, 2478, 2479; Lorenz, in: MünchKommBGB, 5. Aufl. 2008, Vorb. \$474 Rn. 19; Weidenkaff, in: Palandt BGB, 67. Aufl. 2008, \439 Rn. 25; Woitkewitsch VuR 2005, 1, 4 f.; Brömmelmeyer JZ 2006, 493 , 498; Riesenhuber LMK 2006, 199730; Witt NJW 2006, 3322, 3324; a. A. Faust, in: Bamberger-Roth, BGB, 2. Aufl. 2007, $\$ 439$ Rn. 35; MatuscheBeckmann in: Staudinger, BGB, 2004, $₫ 439$ Rn. 56; Fest NJW 2005, 2959, 2961; Gärtner ZGS 2006, 368, 369; Berger, in: Janernig, BGB, 12. Aufl. 2007, \439 Rn. 18

16 BGH NJW 2006, 3200, 3202 Rn. $21 \mathrm{ff}$. - Vorlagebeschluss Quelle: „Diese Bedenken sind auch nach der Auffassung des Senats nicht von der Hand zu weisen."

17 S. etwa EuGH Rs. 120/88, Slg. 1988, 2321 Rn. 23-29 - Mulder; s. vertiefend Everling EuR 1994, 127, $136 \mathrm{ff}$. dere“ und die Aufzählung der Beispiele verdeutliche. ${ }^{18}$ Der Wille des Gesetzgebers, den Verbraucher vor Unannehmlichkeiten zu bewahren, ergebe sich klar aus Art. 3 Abs. 3 Uabs. 3 RiL ${ }^{19}$; Protokollerklärungen hingegen, die in der Richtlinie keinen Anklang gefunden haben, seien insoweit nicht zu berücksichtigen. ${ }^{20}$ Teleologisch sei der Zweck der RiL, ein hohes Verbraucherschutzniveau sicherzustellen. ${ }^{21}$ Systematisch gehe der Hinweis der Bundesregierung auf den 15. Erwägungsgrund der RiL fehl. Satz 1 enthalte gerade keinen allgemeinen Grundsatz, dass die Mitgliedstaaten die Erstattung mindern dürften, weil dieses Recht, wie Satz 2 verdeutliche, vielmehr auf die Vertragslösung begrenzt sei. ${ }^{22}$ Auch rechtsfolgenorientierte Überlegungen ${ }^{23}$ nahm der $E u G H$ in sein Urteil auf: Das Argument der ungerechtfertigten Bereicherung des Verbrauchers gehe ins Leere, weil der Verkäufer gem. Art. 3 Abs. 1 RiL für jede Vertragswidrigkeit hafte. ${ }^{24}$ In der deutschen Literatur war zudem gegenüber dem Bereicherungseinwand vorgetragen worden, dass der Verkäufer trotz der gelieferten mangelhaften Sache mit dem Kaufpreis zu seinem Vorteil arbeiten könne, ohne diese Vorteile herausgeben zu müssen. ${ }^{25}$ Schließlich sei der Verkäufer vor unverhältnismäßigen Kosten geschützt, weil er die Nachlieferung insoweit verweigern könne (hierzu sogleich unter 2.). ${ }^{26}$

Rechtstatsächlich zeigt ein rechtsvergleichender Blick nach Österreich, dass die Umsetzung der RiL im Hinblick auf die Unentgeltlichkeit dort von Anfang an richtlinienkonform erfolgte, denn $\mathbb{\int} 8$ Abs. 3 Konsumentenschutzgesetz ${ }^{27}$ enthält die Pflicht zur kostenlosen Nachbesserung und $\ 932$ ABGB $^{28}$ sieht keinen Nutzungsersatz für den Unternehmer vor. Auch in den anderen Mitgliedstaaten war der Nutzungsersatzanspruch nicht umstritten. ${ }^{29}$

\section{2. Ökonomische Implikationen}

Ob die Entscheidung wirklich den Verbraucherschutz weiter stärkt, kann mit guten Gründen bezweifelt werden. Zwar wird der Verbraucher, dem eine Nutzungsentschädigung droht, seine Mängelgewährleistungsansprüche im Zweifel nicht ausüben. ${ }^{30}$ Vordergründig ist ihm mit der unentgeltlichen Ausübung seiner Rechte nun unmittelbar geholfen, und es ist zu erwarten, dass der Verbraucher nach dem Urteil des $E u G H$ stärker auf die Unentgeltlichkeit der Nacherfüllung achten und seinen Nacherfüllungsanspruch eher wahrnehmen wird.

$18 E u G H$ Rs. C-404/06-Quelle = JZ 2008, 942, 943 Rn. 31.

$19 E u G H$ Rs. C-404/06 - Quelle = JZ 2008, 942, 943 Rn. 35. Vorher schon EuGH Rs. C-292/89, Slg. 1991, I-745 Rn. 18 - Antonissen. Zur historischen Auslegung s. auch Stotz, in: Riesenhuber, Europäische Methodenlehre, 2006, \22 Rn. 15.

20 EuGH Rs. C-404/06 - Quelle = JZ 2008, 942, 943 Rn. 32.

$21 E u G H$ Rs. C-404/06 - Quelle = JZ 2008, 942, 943 Rn. 36. Dies ergibt sich auch aus Erwägungsgrund 1 der RiL.

22 EuGH Rs. C-404/06 - Quelle = JZ 2008, 942, 943 Rn. 39.

23 Zum „effet utile“ als Ausdruck der Rechtsfolgenorientierung s. Deckert, Folgenorientierung in der Rechtsanwendung, 1995, S. 52.

$24 E u G H$ Rs. C-404/06 - Quelle = JZ 2008, 942, 943 f. Rn. 40, 41.

25 Roth JZ 2001, 475, 489; Gsell NJW 2003, 1969, 1970; Kobler ZGS 2004, 48, 49; Wagner/Michael ZGS 2005, 368, 372.

26 EuGH Rs. C-404/06 - Quelle = JZ 2008, 942, 944 Rn. 42.

27 S. http://www.eu-consumer-law.org/legislation84.pdf (abgerufen am 3. 9. 2008)

28 Zum Allgemeinen Bürgerlichen Gesetzbuch s. http://www.eu-consumer-law.org/legislation163.pdf (abgerufen am 3. 9. 2008).

29 S. hierzu die instruktive Studie des Consumer Law Compendium unter http://www.eu-consumer-law.org/study2_de.pdf\#tm00109, S. 728 f. (abgerufen am 3. 9.2008).

30 EuGH Rs. C-404/06 - Quelle = JZ 2008, 942, 943 Rn. 34. Aus der Literatur Roth JZ 2001, 475, 489; Gsell JuS 2006, 203, 204; Rott BB 2004, 2478, 2479; Riesenhuber LMK 2006, 199730. 
Allerdings wirkt dieser gestärkte Rechtsbehelf wie eine Zwangsversicherung gegenüber allen Verbrauchern. Letztlich wird sich nämlich die Risikoerhöhung für den Verkäufer in höheren Preisen der Produkte niederschlagen, die dann voraussichtlich von allen Käufern zu tragen sind. ${ }^{31}$ So ist etwa der finanzielle Aufwand für den Verkäufer enorm, wenn der Käufer nach anderthalb Jahren ein mangelhaftes, gebrauchtes Kfz gegen ein neues, mangelfreies tauschen kann, ohne für die eineinhalbjährige Nutzung zahlen zu müssen. Der EuGH betont in der Entscheidung, dass die Ersatzlieferung abgelehnt werden kann, wenn dies unverhältnismäßig wäre, weil sie unzumutbare Kosten verursachen würde. ${ }^{32}$ In der Literatur wurde schon gemutmaßt, dass das Urteil möglicherweise ein „Danaer-Geschenk“ darstelle, weil der Verkäufer die Ersatzlieferung ablehnen könne. ${ }^{33}$ Allerdings bleibt der Verkäufer weiterhin zur Nachbesserung verpflichtet. $\mathrm{Ob}$ die Beseitigung des Mangels bei technischen Problemen die kostengünstigere Alternative gegenüber der Neulieferung darstellt, darf aber bezweifelt werden. Auch eine Übertragung der schadensrechtlichen Grundsätze „neu

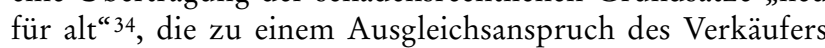
führen würde, verstößt gegen das in der RiL verortete Postulat der „unentgeltlichen“ Nacherfüllung und kann daher nicht für einen Interessenausgleich herangezogen werden. Es bleibt also dabei, dass das Urteil negative wirtschaftliche Auswirkungen auf Unternehmer haben wird, die ihrerseits versuchen werden, ihre Kosten auf alle Kunden umzulegen.

\section{Die Umsetzung des Urteils durch Legislative und Judikative}

\section{Vorgaben des EuGH - die Pflicht zur Gesetzes- korrektur und gemeinschaftskonformen Auslegung}

Schon aus Gründen der Rechtsklarheit ist der deutsche Gesetzgeber gezwungen, den Norminhalt des $\$ 439$ Abs. 4 i. V.m. $\$ 346$ Abs. 1 a.E. BGB bei Verbraucherkaufverträgen zu ändern. Nach ständiger Rechtsprechung des $E u G H$ verlangt die Umsetzung einer Richtlinie, „dass die sich aus diesem Recht ergebende Rechtslage hinreichend bestimmt und klar ist und dass die Begünstigten in die Lage versetzt werden, von allen ihren Rechten Kenntnis zu erlangen und diese gegebenenfalls vor den nationalen Gerichten geltend zu machen ". ${ }^{35}$ Eine richtlinienkonforme Auslegung reicht allein nicht aus, weil diese „nicht die Klarheit und Bestimmtheit aufweisen kann, die notwendig ist, um dem Erfordernis der Rechtssicherheit zu genügen“. ${ }^{36}$ Nicht von ungefähr hat der deutsche Gesetzgeber oft Gesetze nachgebessert, wenn sie für nicht europarechtskonform erklärt wurden. ${ }^{37}$

Allerdings gibt es auch Gegenbeispiele: Trotz der Dietzinger-Entscheidung ${ }^{38}$ wurde in $\$ 312 \mathrm{Abs.} 1 \mathrm{BGB}$ nicht das

31 Zutreffend insoweit Felling MDR 2008, 733, 734

32 EuGH Rs. C-404/06 - Quelle = JZ 2008, 942, 944 Rn. 42 zu Art. 3

Abs. 3 Uab. 2 RiL - umgesetzt in $₫ 439$ Abs. 3 S. 1 BGB.

33 Faust JuS 2008, 652, 653.

34 Gsell NJW 2003, 1969, 1971.

35 EuGH Rs. C-365/93, Slg. 1995, I-499, Rn. 9 - Kommission/Griechenland; Rs. C-144/99, Slg. 2001, I-3541 Rn. 17 - Kommission/Niederlande.

36 EuGH Rs. C-144/99, Slg. 2001, I-3541 Rn. 21 - Kommission/Niederlande.

37 Etwa $\ 611$ a. F. nach $E u G H$ Rs. C-180/95, Slg. 1997, I-2195 - Draehmpaehl oder $\ 912$ Abs. 2 ZPO nach EuGH Rs. C-398/92, Slg. 1994, I-467 Mund \& Fester $=$ JZ 1994, 1165 oder Art. 12a Abs. 4 Satz 2 GG nach EuGH Rs. C-285/98, Slg. 2000, I -69 - Tanja Kreil = JZ 2000, 412 mit Anm. V. Götz.

$38 E u G H$ Rs. C 45/96, Slg. 1998, I-1221 - Dietzinger.
Tatbestandsmerkmal „entgeltlich“ gestrichen. ${ }^{39}$ Die Verpflichtung, das nationale Recht gemeinschaftskonform auszulegen, besteht trotz der Umsetzungspflicht des nationalen Gesetzgebers fort. Sie fordert einerseits keine Rechtsfortbildung „contra legem“, andererseits aber eine richtlinienkonforme Auslegung ,soweit wie möglich“. Wörtlich formuliert der EuGH: „Der Grundsatz der gemeinschaftsrechtskonformen Auslegung verlangt jedoch, dass die nationalen Gerichte unter Berücksichtigung des gesamten nationalen Rechts und unter Anwendung ihrer Auslegungsmethoden alles tun, was in ihre Zuständigkeit liegt, um die volle Wirksamkeit der fraglichen Richtlinie zu gewährleisten und zu einem Ergebnis zu gelangen, das mit dem von der Richtlinie verfolgten Ziel übereinstimmt. “40

\section{Verstoß gegen contra legem-Rechtsprechung?}

Im Vorlagebeschluss betonte der VIII. Senat, nach geltendem Recht stünde dem Verkäufer ein Nutzungsersatzanspruch zu, weil Wortlaut und Wille des Gesetzgebers „eindeutig“ seien. Es würde einen Verstoß gegen die Bindung an Recht und Gesetz gem. Art. 20 Abs. 3 GG darstellen, wenn sich der Richter über den Wortlaut und klar erkennbaren Willen hinwegsetzen würde ${ }^{41}-\operatorname{der} B G H$ zitiert hierfür zahlreiche Entscheidungen des $B \operatorname{Verf} G$. Auch in der Literatur wird die Ansicht vertreten, eine solche Rechtsfortbildung „contra legem" sei nicht zulässig. ${ }^{42}$ Die Gegenansicht bejaht dagegen die Möglichkeit, dass der nationale Richter $₫ 439$ Abs. 4 BGB teleologisch reduziert und damit einen Nutzungsersatzanspruch des Verkäufers in Übereinstimmung mit europäischem Recht verneint. ${ }^{43}$

$\mathrm{Ob}$ hier wirklich eine unzulässige Rechtsfortbildung "contra legem" vorliegt, ist im Folgenden anhand der nationalen Rechtsdogmatik vertieft zu prüfen. Dazu sind die Auslegungsfiguren „Wortlaut, Systematik und Wille des Gesetzgebers" genauer zu untersuchen und vor allem zeitgemäß zu interpretieren.

\section{Die Konkretisierung der richtlinienkonformen Rechtsfortbildung mit den Vorgaben nationaler Rechtsdogmatik}

\section{a) Zum vermeintlich eindeutigen Wortlaut des Gesetzes}

Schon der Hinweis auf den "eindeutigen Wortlaut" ist nur bedingt überzeugend. In der juristischen Methodenlehre ist anerkannt, dass oft schon zweifelhaft ist, ob der Wortlaut des zu prüfenden Tatbestandsmerkmals wirklich so eindeutig ist. Die Feststellung, dass etwas eindeutig ist, setzt notwendigerweise die Auslegung voraus. Das Postulat, nur bei einem mehrdeutigen Wortlaut sei eine weitergehende Auslegung zulässig, gerät damit schnell in die Nähe des Zirkelschlus-

39 Grüneberg, in: Palandt (Fn. 15), $\$ 312$ Rn. 8 spricht von der Verpflichtung zur weiten Auslegung des Begriffs ,entgeltlich“.

40 EuGH Rs. C-212/04, Slg. 2006, I-6057 Rn. 111- Adeneler = JZ 2007, 187 mit Anm. Franzen; verb. Rs. C-397/01 bis C-403/01, Slg. 2004, I-8835 Rn. 114 ff., 118 f. - Pfeiffer.

$41 B G H$ NJW 2006, 3200, 3201 Rn. 15 - Vorlagebeschluss Quelle.

42 Unberath ZEuP 2005, 5, 39; Lorenz, in: MünchKommBGB (Fn. 15), vor $\int 474$ Rn. 19; ders. NJW 2006, 3203; ders. NJW 2007, 1, 6; Witt NJW 2006, 3322, 3324; Fischinger EuZW 2008, 312, 313; Schulze GPR 2008, 128 , 131.

43 Büdenbender, in: Dauner-Lieb/Heidel/Lepa/Ring, Anwaltskommen$\operatorname{tar}$ (Fn. 15), \439 Rn. 43; Schwab JuS 2002, 630, 636; Gsell NJW 2003, 1969 , 1970 f.; Kohler ZGS 2004, 48, 53; im Grundsatz auch Standinger ZJS 2008, 309, 310; Herrler/Tomasic BB 2008, 1245, 1248; Herresthal NJW 2008, 2475, 2477. 
ses. ${ }^{44} \mathrm{Oft}$ wird eine Legaldefinition aus einem Gesetzeszusammenhang herausgerissen und in einen anderen Kontext gestellt, obwohl dies nicht passt. Ein offensichtliches Redaktionsversehen des Gesetzgebers muss korrigiert werden können. Auch Gesetzesumgehungen lassen sich mit der Eindeutigkeitsregel nicht erfassen. Der Sprachgebrauch kann sich wandeln. Und schließlich verengt das Abstellen auf den vermeintlich eindeutigen Wortlaut den im Recht oft erforderlichen Diskurs und überhöht die eigene Ansicht vorschnell als die einzig Richtige. $\mathrm{Zu}$ Recht wird deshalb betont, dass die Eindeutigkeitsregel als bequemes Formalargument die sachliche Begründung abschneidet. ${ }^{45}$ In der Rechtsprechung wird die starre Eindeutigkeitsregel deshalb auch zu Recht abgelehnt. 46

Dass der Wortlaut auch in concreto weniger eindeutig ist als vom $B G H$ behauptet, verdeutlicht schon die Formulierung von $\ 439$ Abs. 4 BGB, wonach der Verkäufer Rückgewähr der mangelhaften Sache nach Maßgabe der $\iint 346-348$ BGB verlangen kann. Weil $\ 346$ Abs. 1 BGB zwischen Rückgabe der Sache und Herausgabe tatsächlicher bzw. pflichtwidrig nicht gezogener Nutzungen unterscheidet, ist zumindest denkbar, dass $\ 439$ Abs. 4 BGB nur auf die erste Alternative des $₫ 346$ Abs. 1 BGB verweist. ${ }^{47}$

Entscheidend ist aber schließlich, dass nach herrschender Ansicht die vom EuGH aufgestellte Verpflichtung zur gemeinschaftskonformen Auslegung neben der richtlinienkonformen Auslegung auch die richtlinienkonforme Rechtsfortbildung umfasst, die über den Wortlaut der Vorschrift hinausgeht. 48 Dies gilt schon deshalb, weil der EuGH unter Auslegung auch die Rechtsfortbildung jenseits der Wortlautgrenze versteht. ${ }^{49}$ Im Einzelnen umfasst die richtlinienkonforme Rechtsfortbildung etwa die Analogie oder auch die teleologische Reduktion, soweit der nationale Richter nicht verfassungsrechtlich oder methodisch daran gehindert ist. Damit kann der Wortlaut für sich genommen der richtlinienkonformen Rechtsfortbildung nicht entgegenstehen.

44 Dworkin, Law's Empire, 1986, S. 352: "The description ,unclear“ is the result rather than the occasion of Herkules' method of interpreting statuory texts." Müller/Christensen, Juristische Methodik I, 9. Aufl. 2004, Rn. 258 sprechen von „deutender Vorwegnahme des möglichen Normsinns“.

45 Von einer unhaltbaren Regel spricht Säcker, in: MünchKommBGB, 5. Aufl. 2006 Einl. Rn. 107; Enneccerus/Nipperdey, Allgemeiner Teil des Bürgerlichen Rechts, 1. Hb. 1959, $\ 56$, S. 333; deutlich auch Kramer, Methodenlehre, 2. Aufl. 2005, S. 73 Fn. 148; Rüthers, Rechtstheorie, 2. Aufl. 2005, Rn. 732: Diese Auffassung ist unhaltbar; zur historischen Herleitung s. Vogenauer, Auslegung von Gesetzen in England und auf dem Kontinent, 2 Bde., 2001, S. 51 ff. A. A. aber Hummel EuZW 2007, 268 (mit unzutreffenden Nachweisen): „Eine auf Grund Ihres Wortlauts nicht auslegungsfähige Rechtsnorm kann auch nach einem Urteil des EuGH immer noch nicht ausgelegt werden."

$46 B G H Z$ 2, 176, 184 - Baugeräte; $B G H Z$ 17, 266, 276 - Magnettonaufnahme; $B \operatorname{Verf} G E$ 1, 415, 416f., 8, 210, 221 - Vaterschaft; $E u G H$ Rs. 6/60, Slg. 1969, 1163, 1194 - Humblet; vgl. auch EuGH Rs. 6/77, Slg. 1977, 1291 Rn. 8/12 - Schouten; Rs. 118/79, Slg. 1988, 607 Rn. 5 - Knauf; Rs. 107/84, Slg. 1985, 2655 Rn. 12 ff. - Befreiung der Post von der Mehrwertsteuer; Rs. C-188/03, Slg. 2005, I-885 Rn. 33 - Junk.

47 So Rott BB 2004, 2478, 2479; ihm folgend OLG Nürnberg NJW 2005, 3000 als Vorinstanz zum $B G H$.

48 Spetzler RIW 1991, 579, $581 \mathrm{f}$, der in diesem Zusammenhang von Analogie spricht; Dänzer-Vanotti RIW 1991, 754, 755; Grundmann ZEuP 1996, 399, 420 ff.; Odersky ZEuP 1998, 485, 486; Möllers EuR 1998, 20, 44 f.; ders., Die Rolle des Rechts im Rahmen der europäischen Integration, 1999, S. 72; Hergenröder, in: Festschrift Zöllner, 1999, S. 1139, 1150 ff.; Canaris, in: Festschrift Bydlinski, 2002, S. 47, 81 ff.; Thüsing ZIP 2004, 2301, 2305 f.; Roth EWS 2005, 385, 393 ff.; Unberath ZEuP 2005, 5, 8; Herresthal, Rechtsfortbildung im europarechtlichen Bezugsrahmen, 2006; S. 5 f., 242; ders. EuZW 2007, 396, 397; Schürnbrand JZ 2007, 910; Langenbucher, Europarechtliche Bezüge des Privatrechts, 2. Aufl. 2008, $\$ 1$ Rn. 90; Standinger ZJS 2008, 309, 310.

49 Möllers EuR 1998, 20, 44; Canaris, in: Festschrift Bydlinski, 2002, S. 47, 81; Franck JR 2004, 45, 48.

\section{b) Der (Umsetzungs-)Wille des Gesetzgebers}

Sodann wurde behauptet, der Wille des Gesetzgebers sei „eindeutig“ und verhindere eine zulässige Rechtsfortbildung. ${ }^{50}$ Auch hier möchte man wiederum Vorsicht anmahnen. Zu Recht hat der BGH betont, dass der deutsche Gesetzgeber den Nutzungsersatz bei Rückgabe einer mangelhaften Sache bewusst regeln wollte. ${ }^{51}$ Nur ist der Wille des Gesetzgebers viel weniger eindeutig als behauptet, weil neben der konkreten Regelungsabsicht der allgemeine Wille des nationalen Gesetzgebers existiert, die RiL korrekt umzusetzen. Ein solcher Umsetzungswille besteht nicht nur hypothetisch, sondern lässt sich auch für den hier strittigen Nutzungsersatzanspruch konkret nachweisen. So formuliert nämlich der Gesetzgeber im Rahmen des Nutzungsersatzanspruches: „Mit der Verbrauchsgüterkaufrichtlinie ist eine derartige Verpflichtung des Verbrauchers (Käufers) vereinbar." 52

Nach einer Ansicht soll nun die konkrete Regelungsabsicht im Fall eines Widerspruchs zum generellen Umsetzungswillen den Vorrang haben. ${ }^{53}$ Die Gegenansicht hält den generellen Umsetzungswillen des Gesetzgebers für ausschlaggebend und vorrangig. ${ }^{54}$ Eine dritte Ansicht kommt zum selben Ergebnis, stellt aber zur Begründung auf den hypothetischen Willen des Gesetzgebers ab und fragt daher, was der Gesetzgeber getan hätte, wenn er seinen Verstoß gegen die Richtlinie bemerkt hätte. ${ }^{55}$

Vorzugswürdig ist die Auffassung, nach der der generelle Umsetzungswille des Gesetzgebers den Vorrang gegenüber der konkreten Regelungsabsicht erhält, soweit der Gesetzgeber unbewusst von der Richtlinie abweicht. Allerdings ist nicht von der bloßen Vermutung des Umsetzungswillens ${ }^{56}$ auszugehen, wenn der Umsetzungswille, wie hier geschehen, positiv geäußert wurde. ${ }^{57}$ In der Rechtsdogmatik ist anerkannt, dass ein Redaktionsversehen durch Gerichte "geheilt“ werden kann: Ein solches Redaktionsversehen besteht herkömmlicherweise dann, wenn der Wortlaut des Gesetzes und der gesetzgeberische Wille auseinanderfallen. ${ }^{58}$ Vergleichbares muss auch für den Fall des Auseinanderfallens von generellem Umsetzungswillen und konkreter Regelungsabsicht gelten. Denn nur so ist gewährleistet, dass der Gesetzgeber seine Pflicht zum gemeinschaftskonformen Han-

$50 B G H$ NJW 2006, 3200 Rn. 13 - Vorlagebeschluss Quelle, zustimmend Witt NJW 2006, 3322, 3324.

$51 B G H$ NJW 2006, 3200 Rn. 13 - Vorlagebeschluss Quelle unter Hinweis auf Begr. RegE BT-Drucks. 14/6040, S. 233.

52 Begr. RegE BT-Drucks. 14/6040, S. 233.

53 Franzen, Privatrechtsangleichung durch die Europäische Gemeinschaft, 1999, S. 312 f.; ders. JZ 2003, 321, 324; Piekenbrock/Schulze WM 2002, 521, 525; Herdegen WM 2005, 1921, 1929, Schürnbrand JZ 2007, 910, 916; Schulze GPR 2008, 128, 131.

54 Grundmann ZEuP 1996, 399, 420; Roth, in: Riesenhuber, Europäische Methodenlehre, 2006, $\mathbb{1} 14 \mathrm{Rn}$. 53. Kritisch, weil noch systematische Überlegungen fordernd, s. Möllers EuR 1998, 20, 44 f.; ders. (Fn. 48), S. 72. Zum weiteren Herresthal WM 2007, 1354; Schulte-Nölke/Busch, in: Festschrift Canaris II, 2007, S. 795, 800 ff.; Herrler/Tomasic BB 2008, 1245, 1248.

55 Rott BB 2004, 2478, 2479; Schulte-Nölke ZGS 2006, 321; Witt NJW 2006, 3322, 3324 Fn. 30 (im Widerspruch zu S. 3324 im Text); Looschelders/ Roth, Juristische Methodik im Prozess der Rechtsanwendung, 1996, S. 227, 298.

56 So im Allgemeinen Gebauer, in: Gebauer/Wiedmann, Zivilrecht unter

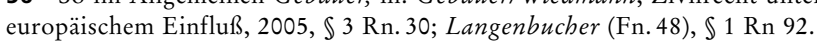
57 Vgl. BT-Drucks. 14/6040, S. 79. Auch im Vertragsrecht muss nur auf den hypothetischen Willen abgestellt werden, soweit der Vertragsinhalt ergänzt werden muss, weil eine Lücke vorliegt, s. Heinrichs, in: Palandt (Fn. 15), \157 Rn. 7.

58 S. Engisch, Einführung in das Juristische Denken, 10. Aufl. 2005, S. 227; für einen rechtsvergleichenden Überblick Vogenauer (Fn. 45), S. 1267. 
deln gemäß $\ 249$ Abs. 3 EGV auch tatsächlich erfüllen kann. Wenn sich der Gesetzgeber im Irrtum befand, also unbewusst von den Vorgaben der Richtlinie abwich, ist es folglich nur in seinem Interesse, wenn die Gerichte diesen Irrtum im Wege der Auslegung korrigieren. ${ }^{59}$ Bei gegenteiliger Behauptung würde man unterstellen, dass der Gesetzgeber sehenden Auges einen Staatshaftungsanspruch wegen unzutreffender Umsetzung riskieren wollte. ${ }^{60}$ Dies wird man den Parlamentariern kaum unterstellen können. Vielmehr wollten sie den Umsetzungsspielraum, der ihnen durch Art. 249 Abs. 3 EGV eingeräumt ist ${ }^{61}$, nutzen, allerdings unter dem Vorbehalt, dass diese Umsetzung dem europäischen Recht entspricht. Würde aber bei Ausnutzung des Gestaltungsspielraums der gemeinschaftsrechtliche Staatshaftungsanspruch drohen, müsste der nationale Gesetzgeber sich künftig viel stärker als in der Vergangenheit sklavisch an die Vorgaben von Richtlinien halten und könnte nicht versuchen, Zweifelsfragen nach seinem Dafürhalten bei der Umsetzung der Richtlinie in nationales Recht zu gestalten. Das durch Rechtsfortbildung gewonnene Ergebnis ist schließlich auch nicht mit einer horizontalen Wirkung der Richtlinie zu vergleichen ${ }^{62}$, denn anders als bei einer direkten Wirkung liegt ein Umsetzungsakt des nationalen Gesetzgebers vor, dessen Ziel die Umsetzung der Richtlinie bildete.

Diese Überlegungen entsprechen im Übrigen auch der deutschen Legislative und Judikative sowie der Rechtsprechung des EuGH: Der XI. Senat des $B G H$ hielt in seinem Heininger-Urteil den generellen Umsetzungswillen für ausschlaggebend. ${ }^{63}$ Der $B G H$ entschied, dass aus dem Irrtum des Gesetzgebers im Hinblick auf die Richtlinienkonformität nicht geschlossen werden könne, dass der Gesetzgeber „sehenden Auges einen Richtlinienverstoß in Kauf genommen habe“. Das Gegenteil sei der Fall und nach dem Willen des Gesetzgebers sollten das nationale Recht und der Richtlinieninhalt übereinstimmen. ${ }^{64}$ Schon vor mehr als 20 Jahren betonte die Bundesregierung, „sich der Notwendigkeit einer richtlinienkonformen Umsetzung bewusst $\mathrm{zu}$ sein“.65 Schließlich geht auch der EuGH davon aus, dass der Mitgliedstaat bei der Umsetzung von Richtlinien in der Absicht handelt, „den sich aus der Richtlinie ergebenden Verpflichtungen in vollem Umfang nachzukommen“.66 Im Ergebnis war damit der Wille des Gesetzgebers weit weniger eindeutig als behauptet.

Etwas anderes kann allerdings dann gelten, wenn sich der deutsche Gesetzgeber bewusst über das europäische Recht hinwegsetzen will. Dies ist, entgegen einzelner Stimmen ${ }^{67}$, nicht etwa graue Theorie: So hatte der deutsche Gesetzgeber bewusst die Vollmachtstreuhand nicht in $\$ 34 \mathrm{a}$ WpHG aufgenommen, obwohl die maßgebliche Richtlinie eine solche Ausgrenzung nicht vorsah. Ein weiteres Beispiel hierfür ist die deutsche Regelung, nach der Rücktritt und Minderung eine erfolglose Fristsetzung zur Nacherfüllung verlangen, obwohl die RiL diese nicht kennt. In diesen Fällen ist eine

59 Rott BB 2004, 2478, 2479.

60 A.A. wohl die Autoren in Fn. 4.

61 Nach Art. 249 Abs. 3 EGV steht den Mitgliedstaaten die Wahl von „Form und Mitteln“ frei.

62 So aber Schürnbrand JZ 2007, 910, 916.

63 BGHZ 150, 248, 257 = NJW 2002, 1881, 1883 Rn. 26 - Heininger.

$64 B G H Z 150,248,257=$ NJW 2002, 1881, 1883 Rn. $26-$ Heininger

65 EuGH Rs. 14/83, Slg. 1984, I-1891 Rn. 11 - von Colson und Kamann.

$66 E u G H$ Rs. C-334/92, Slg. 1993, I-6911 Rn. 20 - Wagner Miret; Rs. C-

397/01 bis C-403/01, Slg. 2004, I-8835 Rn. 112 - Pfeiffer. Mit dieser Über-

legung wird der nationale Richter auch nicht in seiner nationalen Rechts-

dogmatik gebunden, so aber Schürnbrand JZ 2007, 910, 916.

67 So aber Schürnbrand JZ 2007, 910, 916: „praktisch nicht vorkommt“. richtlinienkonforme Rechtsfortbildung nicht möglich, weil sich der Gesetzgeber bewusst gegen die europäische Lösung entschied. ${ }^{68}$ Auch wäre insoweit ein Staatshaftungsanspruch gerechtfertigt. ${ }^{69}$

\section{c) Systematik - Die Richtlinie als Teil der Gesamtrechtsordnung}

Fraglich ist, ob auch systematische Überlegungen die Rechtsfortbildung stützen: In der Literatur wurde herausgearbeitet, dass bei unzureichender Umsetzung einer Richtlinie durch den nationalen Gesetzgeber, der unbewusst hinter dem Regelungszweck der Richtlinie zurückbleibt, nicht nur der deutsche Normtext, sondern auch der europäische Richtlinientext zu den Wertentscheidungen der nationalen Rechtsordnung gehöre. Im Rahmen der Lückenfüllung sei dann die in unvollkommener Form zum Ausdruck gebrachte Entscheidung des nationalen Gesetzgebers entsprechend dem Willen, europarechtskonform zu handeln, zu ergänzen. ${ }^{70}$

Diese Ansicht ist jüngst kritisiert worden: Die Richtlinie sei ein eigenes Regelungsgebäude, ohne lex zu sein. Würde man diesen systematischen Überlegungen folgen, würde der innerstaatliche Normbefehl $\mathrm{zu}$ einer dynamischen Verweisung auf die Richtlinie in ihrer jeweils vom $E u G H$ propagierten Fassung degradiert. ${ }^{71}$ Zudem widerspräche eine solche Auslegung den Grundsätzen des Vertrauensschutzes, insbesondere den Anforderungen an Normenklarheit und Normenbestimmtheit. ${ }^{72}$

Abzustellen ist richtigerweise auf den geäußerten Willen des Gesetzgebers, mit einer konkreten Vorschrift die europäische Richtlinie korrekt umzusetzen. ${ }^{73} \mathrm{Nimmt}$ man diesen Willen ernst, bleibt man in der nationalen Rechtsdogmatik und verhindert auch eine ungehemmte dynamische Verweisung auf den nicht umgesetzten Richtlinientext. Die Wertentscheidung der Richtlinie findet sich damit im Transformationsgesetz wieder. Dann bleibt es bei der schon von $B$ VerfG und $B G H$ postulierten Feststellung, dass die Rechtsfortbildung möglich ist, „wenn die Wertentscheidung, sei es auch nur in unvollkommener Form für eine Lösung in einem bestimmten Sinne zum Ausdruck gebracht wurde“.74 Konsequenterweise ist damit auch nicht gegen den Vertrauensschutz oder Anforderungen an die Normenklarheit verstoßen. Denn jede Rechtsfortbildung bewegt sich vom Wortlaut weg. Wenn der Gesetzgeber sich ausdrücklich auf den Normtext der europäischen Richtlinie beruft, kann der Bürger kaum darauf vertrauen, dass dieser Normtext nicht zur Anwendung kommt. Vielmehr gehen der deutsche Gesetzgeber und damit auch die Bürger das Risiko ein, dass der $E u G H$ den europäischen Normtext kraft seiner Auslegungs-

68 So etwa für $\int 34 \mathrm{a}$ WpHG a.F.s. Möllers, in: Kölner Kommentar zum WpHG (KK), 2007, \34a Rn.39; für das Mängelgewährleistungsrecht, s. Herresthal, in: Langenbucher (Fn. 48), $\ 2$ Rn. 161a.

69 So etwa für $₫ 34$ a WpHG a.F. S. Möllers, in: KK-WpHG (Fn. 68), $\$ 34 a$ Rn. 43.

70 Möllers EuR 1998, 20, 45; ders. (Fn. 48), S. 72 f.; ihm folgend Schnorbus AcP 201 (2001), 860, 896; ebenso Hergenröder, in: Festschrift Zöllner, 1999 , S. 1139, 1145 ff.; Canaris, in: Festschrift Bydlinski, 2002, S. 47, 85 f.; Unberath ZEuP 2005, 5, 8; Roth EWS 2005, 385, 394; Franzen (Fn. 53), S. 416, 419; Langenbucher, in: ders. (Fn. 48), $\ 1$ Rn 92.

71 Herdegen WM 2005, 1921, 1928 f.; Schürnbrand JZ 2007, 910, 913; Lorenz, in: MünchKommBGB (Fn. 15), Vor $\ 474$ Rn. 5; Schulze GPR 2008, $128,131$.

72 Herdegen WM 2005, 1921, 1929; Schürnbrand JZ 2007, 910, 916.

73 Begr. RegE BT-Drucks. 14/6040, S. 233.

$74 B \operatorname{VerfGE~34,~269,~} 287$ = JZ 1973, 662 mit Anm. Kübler; BGHZ 108, 305, 308; BGH NJW 1994, 852, 855; hierzu Möllers EuR 1998, 20, 45; ders. (Fn. 48), S. 72 f.; ihm folgend Schnorbus AcP 201 (2001), 860, 896. 
kompetenz konkretisiert. Die Richtlinie ist auch bindendes Recht: Sie bindet den Mitgliedstaat und seine Organe ${ }^{75}$ und konsequenterweise hat der $E u G H$ auch eine vertikale Drittwirkung von Richtlinien bejaht. ${ }^{76}$ Wie bei der verfassungskonformen Auslegung ist eine richtlinienkonforme Auslegung „im Lichte“77 der Richtlinien dann möglich, wenn der nationale Gesetzgeber die Wertentscheidungen bewusst in das nationale Recht aufnehmen wollte. Dieselben Erwägungen müssen auch für die richtlinienkonforme Rechtsfortbildung gelten.

\section{d) Telos}

Damit sind auch die teleologischen Überlegungen für die gemeinschaftskonforme Auslegung vorgegeben. Diese zielen nicht etwa auf die Analogie als Lückenfüllung des nationalen Rechts, weil es nicht um die Herstellung von Gleichbehandlung, sondern um die Übernahme eines unvollständig umgesetzten Richtlinientextes geht. ${ }^{78}$ Der Zweck der Auslegung ist es, wie der $E u G H$ formuliert, „zu einem Ergebnis zu gelangen, das mit dem von der Richtlinie verfolgten Ziel übereinstimmt“.79

\section{Rechtsfortbildung im Fall der Quelle-Entscheidung}

Im Ergebnis ist damit eine teleologische Reduktion der $\$ \int 439$ Abs. 4 i.V. mit 346 Abs. 1 a.E., Abs. 2 Nr. 1 BGB mit den bisherigen nationalen Auslegungsfiguren vereinbar. Grundsätzlich ist auch eine gespaltene Auslegung möglich (s. sogleich V.). Aber selbst bei Ablehnung der gespaltenen Auslegung käme es nicht zu einem vollständigen Funktionsverlust der Norm, der teilweise als Grenze der gemeinschaftskonformen Rechtsgewinnung 80 bezeichnet wird. Ausgeschlossen würde nämlich lediglich die Herausgabe von Nutzungen, nicht hingegen die Rückgewähr der Sache als solcher. Der überwiegende Teil des Anwendungsbereichs des $\ 439$ Abs. 4 BGB bliebe also bestehen.

75 Nach Art. 249 Abs. 3 EGV ist die Richtlinie: „für jeden Mitgliedstaat (...) verbindlich".

$76 E u G H$ Rs. 148/78, Slg. 1979, 1629 Rn. 22, f. - Ratti; Rs. 8/81, Slg. 1982, 53 Rn. 25 - Becker; Rs. C-201/02, Slg. 2004, I-723 Rn. 57 - Wells.

$77 E u G H$ Rs. 14/83, Slg. 1984, 1891 Rn. 26, 28 - von Colson und Kamann; Rs. C-106/89, Slg. 1990, I-4135 Rn. 8 - Marleasing; BGHZ 150, 248, 252 - Heininger.

78 Auer NJW 2007, 1106, 1108.

$79 E u G H$ Rs. C-212/04, Slg. 2006, I-6057 Rn. 111; Adeneler = JZ 2007 187 mit Anm. Franzen; verb. Rs. C-397/01 bis C-403/01, Slg. 2004, I-8835 Rn. 115 f., 118 f. - Pfeiffer. Einen anderen Weg geht Herresthal (Fn. 48), S. 321 ff.; ders. EuZW 2007, 396, 400; ders. NJW 2008, 2475, 2477. Er lehnt den Gesamtzusammenhang ab, möchte aber den objektivierten Regelungszweck darin sehen, dass die Judikative die ursprüngliche Bewertung des nationalen Gesetzgebers nicht durch ihre eigene Bewertung ersetze, sondern durch die Bewertung des EuGH. Ungeklärt ist damit aber, ob dieser objektivierte Regelungszweck auch rückwirkend für den konkreten Fall oder nur für zukünftige Fälle gilt.

80 BGHZ 150, 248, 259 = NJW 2002, 1881, 1883 - Heininger; Canaris, in Festschrift Bydlinski, 2002, S. 47, 94; Gebauer, in: Gebauer/Wiedmann

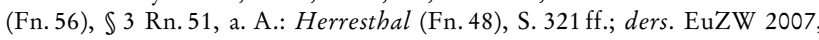
396,400 .
An dieser Stelle ging es nur um die teleologische Reduktion, also die negative Funktion der RiL, nämlich den Ausschluss eines Nutzungsersatzanspruchs. ${ }^{81}$ Andere Fälle sollen an dieser Stelle nicht weiter vertieft werden, etwa die Frage, ob die richtlinienkonforme Auslegung auch einen positiven Anspruch begründen kann oder ob auf bestimmte Tatbestandsmerkmale, wie etwa das Verschulden, verzichtet werden kann. ${ }^{82}$

\section{Zusammenfassung und Ausblick}

Der Gesetzgeber hat den Wortlaut des $\ 439$ Abs. 4 BGB umgehend den Vorgaben der Quelle-Entscheidung anzupassen.

Daneben hat der VIII. Senat als Folge richtlinienkonformer Rechtsfortbildung das Recht $\mathrm{zu}$ einer teleologischen Reduktion des $₫ 439$ Abs. 4 BGB, da diese mit der nationalen Rechtsdogmatik, wie Wortlaut, dem Willen des Gesetzgebers und der Systematik, im Einklang steht. Eine solche Rechtsmethodik entspräche auch der Rechtsprechung des XI. Senates in der Heininger-Entscheidung, der ebenfalls betont, dass der generelle Umsetzungswille dem konkreten Regelungsziel vorgeht. ${ }^{83}$ Schon früher hatte der $B F H$ eine teleologische Reduktion bejaht, als es um die richtlinienkonforme Auslegung eines vermeintlich europarechtskonformen Gesetzes ging. ${ }^{84}$ Will der VIII. Senat aber von den Grundsätzen des XI. Senates abweichen, könnte an die Einberufung des Großen Senats zu denken sein. ${ }^{85}$

Möglich erscheint auch eine gespaltene Auslegung86 von \439 Abs. 4 BGB, so dass eine Nutzungsentschädigung durch den Käufer bei einer Ersatzlieferung nur ausgeschlossen ist, wenn es sich bei Käufer und Verkäufer um Verbraucher und Unternehmer handelt. Eine richtlinienkonforme Reduktion erfolgt danach also nur in den Fällen, die in den Anwendungsbereich der Richtlinie fallen. In allen anderen Fällen bleibt aufgrund der gespaltenen Auslegung eine Nutzungsentschädigung weiterhin möglich. Die historische Wertung des Gesetzgebers würde damit so weit als möglich berücksichtigt.

81 Angesprochen bei Staudinger ZJS 2008, 309, 310

82 Hierzu etwa $B A G E$ 82, 211, $230=$ NJW 1996, 2529, 2533 zum Verschulden bei $\$ 823$ BGB; Canaris, in: Festschrift Bydlinski, 2002, S. 47, 102. Im öffentlichen Recht tut man sich deutlich leichter, etwa die Voraussetzung des $₫ 48$ VwVfG zu derogieren, s. EuGH Rs. C-24/95, Slg. 1997, I-1591, 47 Rn.24f., 38 - Alcan = JZ 1997, 722 mit Anm. Classen, einerseits und $B$ VerfG NJW 2000, 2015 andererseits; hierzu Maurer, Allgemeines Verwaltungsrecht, 16. Aufl. 2006, $\$ 11$ Rn. 38a ff.

83 BGHZ 150, 248, 257 = NJW 2002, 1881, 1883 Rn. 26 - Heininger.

84 BFHE 132, 319, 321 = WM 1981, 444, 445.

85 Siehe $\mathbb{1} 132$ Abs. 2, Abs. 4 GVG: Kissel/Mayer, Gerichtsverfassungsgesetz, 5. Aufl. 2008, $\$ 132 \mathrm{Rn} 15 \mathrm{f} ., \mathrm{Rn} .31 \mathrm{ff}$.

86 Für eine gespaltene Auslegung bei $₫ 439$ Abs. 4 BGB auch Witt NJW 2006, 3322, 3325; Staudinger ZJS 2008, 309, 310. 